Research Article

\title{
Cross-sectional Study on Sero-Prevalence of SARS-CoV-2 Infection in Jabalpur, Madhya Pradesh, India
}

\author{
Sudarshan Ramaswamy ${ }^{2}$, Aditya Athotra', PK Kasar ${ }^{3}$, Rajesh Tiwari ${ }^{3}$, Shashi Prabha \\ Tomar $^{3}$, Ratnesh Kuraria ${ }^{3}$, Riti Jain Seth ${ }^{3}$, Arvind Rai', Meera Dhuria', Arti Bahl', \\ Anil Digambar Patil', SK Jain', $\underline{\text { Sujeet K Singh' }}$
}

${ }^{1}$ National Centre for Disease Control, Delhi, India.

${ }^{2}$ World Heath Organization (WHO) Country Office, Delhi, India.

${ }^{3}$ Government Medical College, Jabalpur, Madhya Pradesh, India.

DOI: https://doi.org/10.24321/0019.5138.202114

I $\quad \mathbf{N} \quad \mathbf{F} \quad \mathbf{O}$

\section{Corresponding Author:}

Shashi Prabha Tomar, Government Medical

College, Jabalpur, Madhya Pradesh, India.

E-mail Id:

nscbmcjb@gmail.com

Orcid Id:

https://orcid.org/0000-0002-7966-4066

How to cite this article:

Ramaswamy S, Athotra A, Kasar PK, Tiwari R, Tomar SP, Kuraria R et al. Cross-sectional Study on Sero-Prevalence of SARS-CoV-2 Infection in Jabalpur, Madhya Pradesh, India. J Commun Dis 2021; 53(1): 82-88.

Date of Submission: 2021-02-10

Date of Acceptance: 2021-03-03
$\begin{array}{llllllll}\mathbf{A} & \mathbf{B} & \mathbf{S} & \mathbf{T} & \mathbf{R} & \mathbf{A} & \mathbf{C} & \mathbf{T}\end{array}$

Introduction: In India, laboratory diagnosis of SARS - CoV-2 infection has been mostly based on real-time reverse transcriptase polymerase chain reaction (RT-PCR). Studies have shown that Viral titres peak within the first week of symptoms, but may decline post this time frame, thereby hampering RT-PCR based diagnostic strategies. These reasons have prompted the call for adoption of antibody testing as a potential source of data to address the gap in data and inform public health and governance policies oriented towards COVID-19.

Materials and Methods: A Cross-sectional study with a sample size of 9000 was conducted for 11 days (Dec 11-21, 2020) including all the 79 wards under Jabalpur Municipal Corporation. Serum samples were tested for the presence of specific antibodies to COVID19 using ICMRKavach IgG ELISA kits. The data collected was compiled on Microsoft Excel and data analysis was carried out using STATA 15E statistical software.

Result: Overall seroprevalence of the study population was found to be $28.70 \%$ (weighted). Wards of the city of Jabalpur were classified into three categories based on the case prevalence - High (27 wards), Medium (26 wards) and Low (26 wards). Based on the overall seroprevalence, the estimated number of total infections were calculated to be $3,54,870$ for the study population. Overall Case Infection Ratio was 31.41.

Conclusion: The current seroprevalence study rightly provides information on proportion of the population exposed, however, the correlation between presence and absence of antibodies is not a marker of total or partial immunity. Hence, the golden rule of Social distancing, sanitization, personal protective equipment and public health measures have to be continued.

Keywords: Jabalpur, Seroprevalence, SARS-CoV-2, Community based, Infections 


\section{Introduction}

Ever since the first case first case of novel Corona Virus infection reported on December 312019 in Wuhan, China, the virus has spread across the globe rapidly and has caused massive catastrophe in various forms. ${ }^{1}$ In the wake of the swift increase in the number of COVID-19 cases worldwide, a global pandemic was declared by the World Health Organisation (WHO) on March 12 2020. ${ }^{2}$ Aggressive interventional efforts have been undertaken worldwide throughout the year 2020 to contain the spread of this health emergency of international concern. Despite intensive containment efforts, as of Feb 6 2021, cumulative cases worldwide have reached more than 106 million with more than 2.3 million deaths. ${ }^{3}$ India being no exception, is one of the worst affected country currently with over one crore people infected and 1.5 lakh deaths. ${ }^{4}$ Containment efforts by India during the year 2020 have mostly relied on mass quarantine or 'lock-down' measures to control and restrict population movement, thereby reducing personto-person contacts. ${ }^{5}$ However, several serological studies, ${ }^{6}$ 7 estimating anti-SARS-CoV-2 antibodies have showed that the actual number of infected individuals are higher than the reported cases due to various factors (Host, Agent and Environmental). ${ }^{8,9}$ Additionally, the requirement for specialist equipment and reagents for sample collection, skilled and trained laboratory staff, unavoidable time-gap between sampling and generation of test results amplify the gap between the reported cases and actual prevalence of infection. In India, laboratory diagnosis of infection has been mostly based on real-time reverse transcriptase polymerase chain reaction (RT-PCR). Furthermore, the issues of quality/timing of collection, transportation time of the samples have impact on the outcomes of RT-PCR. Hence, estimating the seroprevalence status of the community regularly becomes an important catalyst to address the gap in data, thereby informing public health authorities and aiding governance policies oriented towards COVID-19.

Since the announcement of the efficacy of few COVID-19 vaccines through the interim analysis of placebo controlled phase 3 trials (mid phase), various countries including India intensified national deployment plans for COVID-19 vaccine introduction. ${ }^{10}$ Knowing the serological status, Clinicosocial and geographical risk profile in the community for SARS-CoV-2 before vaccine introduction can potentially be helpful in guiding the vaccination program as well. With this backdrop, during the month of December 2020, a seroprevalence study was planned in one of the most affected district (Jabalpur) of the state Madhya Pradesh. Antibody is a protective protein produced by immune system in response to SARS-CoV-2 specific 'antigen'. ${ }^{11}$ IgG antibody generally start appearing after two weeks of the onset of the infection, once the individual has recovered after infection and last for several months. Antibody-based tests would be capable of detecting both ongoing as well as past SARS-CoV-2 infections due to their capacity to detect IgG and IgM, thereby providing valuable clues about asymptomatic infections in the community. ${ }^{12}$ India has indigenously developed its own IgG-based ELISA test for COVID-19, with a reported sensitivity of $92.37 \%$ and a specificity of $97.9 \% .{ }^{13}$ Using this test kit, the following study was conducted among the general population of Jabalpur jointly by department of Community Medicine and Microbiology, Government Medical College, Jabalpur and technical supervision from National Centre for Disease Control, Delhi.

\section{Materials and Methods}

A Cross-sectional study was conducted for 11 days (December, 2020) including all the 79 wards under Jabalpur Municipal Corporation. With due precautions taken to avoid the risk of Covid infection exposure to the participants, a House to House survey was conducted with the help of Data collection team which includes a Lab technician for sample collection and a Nursing Staff for Form filling. During the visit, all participants had to answer a short pre validated questionnaire, translated in their local language. It was followed by the collection of a blood sample by a trained member the team. All individuals aged 1 to less than 18 years for whom parents or guardian are willing to give assent and any individual who is 18 years and above and is ready to give consent for participation were included in the study. Only those who have been residing in Jabalpur since March 1, 2020 were included. Individuals who did not give consent or who did not respond were excluded from the study. Individuals not in compos mentis or those who were intoxicated too were excluded.

As provided by JMC Jabalpur, the population of the city $12,06,219$ (As provided by CMHO Jabalpur) was considered as the Target Population. In May 2020 protocol of ICMR, the sero-prevalence in hotspot areas was considered as $5 \% .{ }^{14}$ Hypothesizing $1 \%$ rise each for June to October and $0.5 \%$ for $1^{\text {st }}$ half of November, it is taken as $10.5 \%$. This prevalence was arbitrarily used for estimating the Sample size in this study. With Confidence Level of 95\%, Absolute Precision of 1\% and Design Effect of 2.5 the effective sample size was calculated using OpenEpi and was approximated to 9000 . Stratified Random Sampling method was used to collect the samples and the required sample was divided proportionally across 79 wards under Jabalpur Municipal Corporation according to their population weightage. Population weighted sample size was calculated for each ward and proportionate to the population of each ward, the number of samples were collected. Computer generated randomized household addresses were availed for the required sample size plus $20 \%$ considering attrition and non-consent/ non-response. 
The urban area of Jabalpur was stratified based on wardwise geospatial mapping. Each ward was considered as one Strata. Kish Grid method was used to randomly identify each candidate based on age and gender. Considering the fact that sex-ratio of Jabalpur is 925 females per 1000 males15, almost similar representation was given to males and females. Furthermore, considering the fact that children and adolescents (aged 1 to less than 18 years) represent an important cohort of the society and previous studies emphasizing the fact that children are just as likely as adults to be infected with this virus but less likely to be symptomatic or develop severe symptoms. ${ }^{16,17}$ There is an urgent need to gather evidence of this under-represented population, hence the sample population age groups were divided into three namely: $33 \%$ males of age 18 years and above, $33 \%$ females of age 18 years and above, $33 \%$ children and adolescent aged 1 to 18 years.

After securely packing the collected samples in a temperature controlled insulated boxes for maintaining the integrity, they were carried back to the field office immediately. The blood samples were stored in the site offices within specialised refrigerators at $40 \mathrm{C}$ before being further processed. Each blood sample was stored for up to one month at the mentioned temperature. After processing of the blood samples, the remnants were discarded taking all biohazard safety precautions. Blood samples thus collected and transported by maintaining Cold chain were processed as follows:

- The samples were de-identified, and testing was performed in an anonymous manner

- The serum was separated by using centrifugation at 2200-2500 rpm for 20 minutes

- Aliquots of serum samples were preserved for batch testing and repeated freeze and thaw cycles were avoided

- Serum samples were tested for the presence of specific antibodies to COVID19 using ICMR-Kavach IgG ELISA kits

- Simultaneously, dry blood on pre-circled filter paper were collected from $15 \%$ of 9000 samples for parallel testing using same ICMR-Kavach IgG ELISA for comparing the results of test using venous blood $\mathrm{v} / \mathrm{s}$ dried blood spot (with an intention that could help in deciding about mode of sample collection in future surveys)

- Interpretation of sero-reactivity was done as per the criteria provided by the manufacturer

This real time monitoring was done from specially designated Covid Control Room to resolve any issues, problems, discrepancies, and technicalities faced by field teams. Parallel coordination with various stakeholders such as Jabalpur District Administration, Health Department and NCDC (National Centre for Disease Control, Delhi) was carried out.

Informed written consent was taken from the potential participants after getting verbal consent for participation in the study. In case of adults, informed written consent was obtained from the participant and in case of adolescents informed written assent was obtained. For children below 12 years, consent was obtained from their parents/ legal guardians. Relevant ethical clearance will be taken from participating institutions (Institutional Ethical Committee) prior to the initiation of the study. After completion of data collection, the data from each field team was compiled on Microsoft Excel and data analysis was carried out using appropriate statistical software.

\section{Result}

In the study, overall 9,279 blood samples surveyed were found to be satisfactory as per lab standards and results of these samples have been utilized for estimation of weighted seroprevalence of SARS-CoV2 antibodies in general population of Jabalpur. Overall 2,665 samples showed positive results and thus the weighted seroprevalence was estimated to be $28.7 \%(\mathrm{Cl}: 27.81-$ $29.63 \%)$. Subsequently, adjusted seroprevalence as per sensitivity and specificity of ELISA test kit was calculated and was found to be $29.42 \%$ (Cl: $28.41-30.43 \%)$. Data collected using a predesigned questionnaire contained basic socio-demographic parameters as well. It was used to analyse the gender wise distribution, age group wise distribution of seroprevalence and socio-economic groups, weighted and adjusted seroprevalence thus calculated is as shown in Table 1.

Table I.Seroprevalence of SARS-CoV-2 antibodies by age, gender and socio-economic profile

\begin{tabular}{|c|c|c|c|c|c|}
\hline Category & Variable & $\begin{array}{c}\text { Samples } \\
\text { collected }\end{array}$ & $\begin{array}{c}\text { Samples tested } \\
\text { positive }\end{array}$ & $\begin{array}{c}\text { Weighted } \\
\text { prevalence }\end{array}$ & $\begin{array}{c}\text { Adjusted prevalence* } \\
(95 \% \mathbf{C l})\end{array}$ \\
\hline \multirow{2}{*}{ Gender } & Male & 3629 & 1119 & $30.83 \%$ & $31.77 \%(30.11-33.43)$ \\
\cline { 2 - 6 } & Female & 5650 & 1546 & $27.36 \%$ & $27.91 \%(26.63-29.19)$ \\
\hline \multirow{2}{*}{$\begin{array}{c}\text { Age group } \\
\text { (years) }\end{array}$} & $0-18$ & 1023 & 282 & $27.51 \%$ & $28.07 \%(25.03-31.10)$ \\
\cline { 2 - 6 } & $19-45$ & 5413 & 1506 & $27.83 \%$ & $28.43 \%(27.12-29.74)$ \\
\cline { 2 - 6 } & $46-60$ & 1875 & 601 & $32.05 \%$ & $33.13 \%(30.79-35.47)$ \\
\cline { 2 - 6 } & $>60$ & 968 & 276 & $28.51 \%$ & $29.19 \%(26.02-32.34)$ \\
\hline
\end{tabular}




\begin{tabular}{|c|c|c|c|c|c|}
\hline \multirow{3}{*}{$\begin{array}{c}\text { Socio } \\
\text { economic } \\
\text { group }\end{array}$} & Non-slum & 7383 & 2116 & $28.66 \%$ & $29.35 \%(28.22-30.48)$ \\
\cline { 2 - 6 } & Slum & 1896 & 546 & $28.95 \%$ & $29.68 \%(27.43-31.93)$ \\
\cline { 2 - 6 } & BPL & 4398 & 1239 & $28.77 \%$ & $29.48 \%(27.34-30.27)$ \\
\cline { 2 - 6 } & APL & 4881 & 1426 & $29.21 \%$ & $29.97 \%(28.55-31.38)$ \\
\hline \multicolumn{2}{|c|}{ Overall } & 9279 & 2665 & $28.72 \%$ & $29.42 \%(28.41-30.43)$ \\
\hline
\end{tabular}

BPL - Below Poverty Line18, APL - Above Poverty Line.

Wards of the city were arranged in a sequential order as per case prevalence (cumulative reported cases/ 10,000 population) and classified into three categories (tertiles) High (27 wards), Medium (26 wards) and Low (26 wards). Based on this classification of wards, status of SARS-CoV-2 antibody seroprevalence has been described in Table 2 . No significant difference in seroprevalence was observed in different categories of wards as per the prevalence of cumulative cases reported. However, it was observed that there was a wide range of difference between individual wards across the city. For example, wards such as Hanuman Tal ward, Shitla Mai Ward showed a seroprevalence of
43.39\% and $42.30 \%$ respectively whereas wards like Swami Dayanad Saraswati Ward and Dr. Jakir Hussain Ward showed seroprevalence as low as $12.74 \%$ and $13.13 \%$ respectively. A seroprevalence of $\geq 40 \%$ was observed in 6 wards and $\leq 20 \%$ was observed in 14 wards. Wards of the city were arranged in a sequential order as per seroprevalence and classified into three categories (tertiles) - High (27 wards), Medium (26 wards) and Low (26 wards) and the details are as shown in Table 2. Pictorial representation of the city wards categorized by case prevalence and seroprevalence has been depicted in the form of Heatmaps for comparison, as in Figure 1.

Table 2.Seroprevalence of SARS-CoV-2 antibodies by wards (low, medium and high - case burden and seroprevalence)

\begin{tabular}{|c|c|c|c|c|c|c|}
\hline Category & Tertile & $\begin{array}{l}\text { Average cases } \\
\text { reported per } 1 \\
\text { lakh population }\end{array}$ & $\begin{array}{c}\text { Total } \\
\text { samples } \\
\text { collected }\end{array}$ & $\begin{array}{c}\text { Samples } \\
\text { tested } \\
\text { positives }\end{array}$ & $\begin{array}{l}\text { Weighted } \\
\text { prevalence }\end{array}$ & $\begin{array}{c}\text { Adjusted } \\
\text { prevalence } \\
(95 \% \mathrm{CI})\end{array}$ \\
\hline \multirow{3}{*}{$\begin{array}{c}\text { Wards } \\
\text { categorized by } \\
\text { case prevalence }\end{array}$} & High & 1769 & 3195 & 925 & $28.64 \%$ & $29.45 \%(29.38-29.53)$ \\
\hline & Moderate & 755 & 2863 & 842 & $29.68 \%$ & $30.59 \%(30.53-30.66)$ \\
\hline & Low & 316 & 3221 & 898 & $28.90 \%$ & $28.82 \%(28.75-28.89)$ \\
\hline \multirow{3}{*}{$\begin{array}{l}\text { Wards } \\
\text { categorized by } \\
\text { sero-prevalence }\end{array}$} & High & 954 & 3118 & 1150 & $36.91 \%$ & $41.08 \%(41.05-41.11)$ \\
\hline & Moderate & 964 & 3007 & 872 & $29.03 \%$ & $32.31 \%(32.29-32.32)$ \\
\hline & Low & 899 & 3154 & 643 & $20.31 \%$ & $22.59 \%(22.56-22.63)$ \\
\hline \multicolumn{2}{|c|}{ Overall } & 939 & 9279 & 2665 & $28.72 \%$ & $29.42 \%(28.41-30.43)$ \\
\hline
\end{tabular}
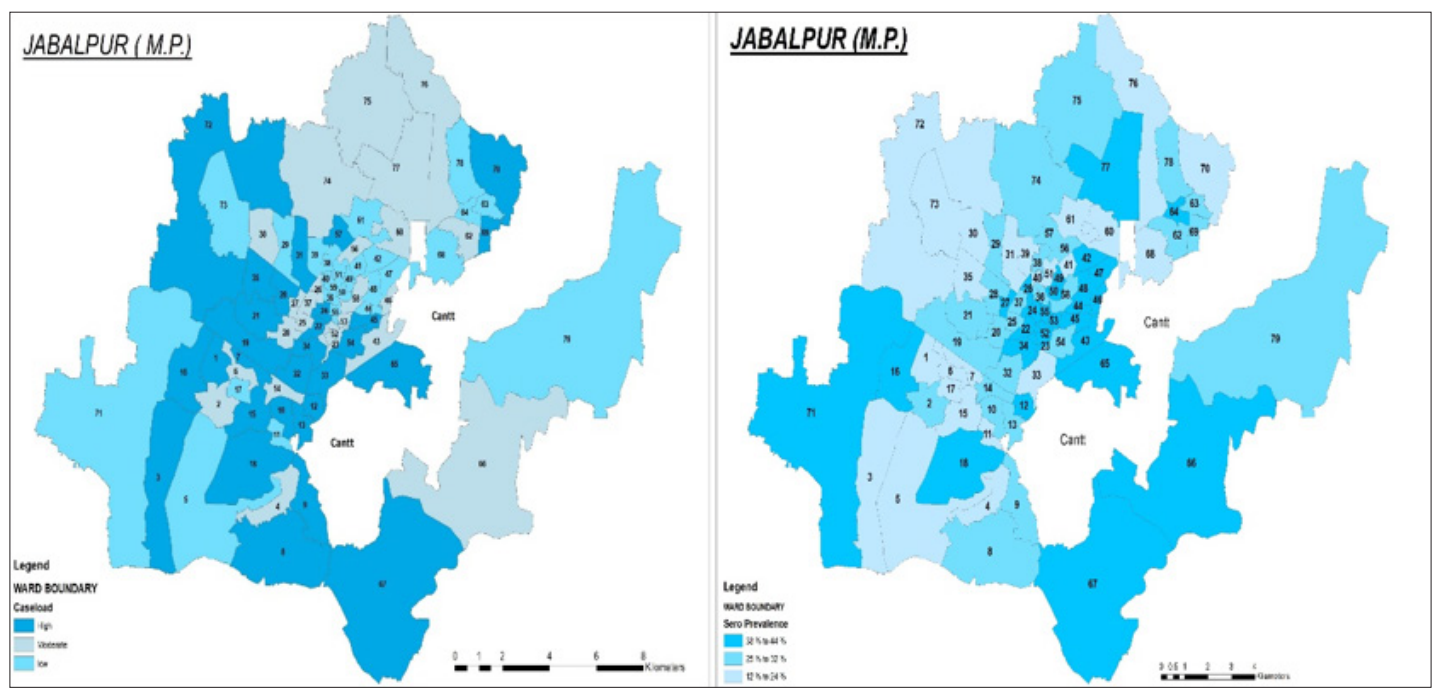

Figure I.Heat map of Jabalpur city Covid I 9 Case prevalence as on Dec I, 2020 (Left) and SARS-CoV-2 antibodies Sero-prevalence (Right) 
Table 3.Infection case ratio based on wards categorized according to SARS-CoV-2 seroprevalence

\begin{tabular}{|c|c|c|c|c|c|}
\hline $\begin{array}{c}\text { Risk category based on } \\
\text { sero- prevalence }\end{array}$ & $\begin{array}{c}\text { Population } \\
\text { (Percentage) }\end{array}$ & $\begin{array}{c}\text { Adjusted sero- } \\
\text { prevalence }\end{array}$ & $\begin{array}{c}\text { Positive cases as } \\
\text { on Dec 1, 2020 }\end{array}$ & $\begin{array}{c}\text { Expected } \\
\text { infections }\end{array}$ & $\begin{array}{c}\text { Infection- } \\
\text { case ratio }\end{array}$ \\
\hline High & $4,05,561(33.92 \%)$ & $41.08 \%$ & 3,867 & $1,66,592$ & 43.08 \\
\hline Moderate & $3,91,537(32.46 \%)$ & $32.31 \%$ & 3,821 & $1,26,487$ & 33.10 \\
\hline Low & $4,09,121(33.62 \%)$ & $22.59 \%$ & 3,611 & 92,426 & 25.60 \\
\hline Overall & $12,06,219(100 \%)$ & $29.42 \%$ & 11,299 & $3,54,870$ & 31.41 \\
\hline
\end{tabular}

Based on the overall adjusted seroprevalence, the estimated number of total infections were calculated to be $3,54,870$ for the study population with Infection-Case Ratio (ICR) of 31.41. Details of estimated infection and ICR among wards categorized based on seroprevalence is depicted in Table 3.

\section{Discussion}

Overall weighted seroprevalence of the study population was found to be $28.72 \%$ and the adjusted seroprevalence was $29.42 \%$. Seroprevalence studies across the world show a wide spectrum of seroprevalence based on different stages of the epidemic. Findings from similar studies have been reported from Iran, United States and Germany with seroprevalence of $22 \%, 21-30 \%$ and $15 \%$ respectively. ${ }^{19-21}$ There has been a wide range of reports on seroprevalence of SARS-CoV2 anti bodies from various places across India as well, including $57 \%$ in Urban Slums of Mumbai to $23.5 \%$ among general population of Delhi. ${ }^{22,23} \mathrm{~A}$ nationwide serosurvey conducted during Dec 2020 by ICMR estimates that the overall seroprevalence in India is $21.4 \%$, seroprevalence of $31.7 \%$ in urban slums and $26.2 \%$ in urban non-slums. ${ }^{7}$ Our study conducted in Jabalpur during the same period is consistent with the national serosurvey results as well.

In our study, irrespective of the average cases reported per 1lakh population as on Dec 12020 (Mid Survey date when $50 \%$ of samples were collected within 14 days incubation period), there was no significant difference in seroprevalence (Table 2). High, medium and low case prevalent category (tertiles) of wards had no significant difference in seroprevalence. A similar study conducted in Indore (7.75\% overall seroprevalence) during August showed the seroprevalence was consistent with case prevalence. ${ }^{24}$ This relation is not observed in our study since the spread of disease is more extensive, indicated by the overall seroprevalence of Jabalpur in December is 4 times that of Indore in August. As the disease spreads, it can be hypothesized that the differences even out and there shall be more uniformity in disease spread. This can also be seen consistent with the fact the hotspot areas were 5 times more affected than the low seroprevalent wards in the Indore study as compared to our study where seroprevalence of hotspot areas was three times higher than the low risk areas. Case prevalence and seroprevalence always do not follow a linear relationship which is also supported by a SEROCoV-POP prospective study conducted in Switzerland which reported that most of the population of Geneva remained uninfected during the wave of the pandemic between April -May 2020, despite the high prevalence of COVID-19 in the region. ${ }^{25}$ However, the SEROCoV-POP prospective study showed a significant difference in seroprevalence among different age groups where as our study shows no significant association between seroprevalence and age groups or gender or socio-economic groups. The pattern indicates that the spread of disease becomes more even as the disease progresses indicating that the proportion of susceptible population if higher in any group, they have higher chance of getting infected over time and hence such scenario could be noticed.

The overall Infection-Case Ratio in our study for Jabalpur was 31.41. Infection-case ratio calculated as per recent ICMR serosurvey result of $21.4 \%$ was 27 and our study results are in accordance with the national figures. However, the difference in ICR between different tertiles indicate the variation in case detection/ reporting and effectiveness of contact tracing program in different wards. Low seroprevalent areas with high case reporting are the best performing wards and high seroprevalent areas with low case reporting are the worst performing wards (Figure 1). As the seroprevalence rises, the proportion of susceptible population decreases and thus as the epidemic progresses, the variation in ICR should reduce. Repeated serosurveys and comparative studies shall aid in surveillance strategies and identifying the key aspects where improvement in intervention planning and implementation is essential. Lower the ICR, better is the surveillance program as higher proportion of asymptomatic cases get detected through effective contact tracing.

The current seroprevalence study rightly provides information on proportion of the population exposed, nevertheless the correlation between presence and absence of antibodies is not a marker of total or partial immunity. It must also be noted that more than 70 percent of the population is still susceptible for COVID-19 infection. From the study it's also clear that since the disease in spreading 
more evenly among all age groups, socio-economic groups with no predisposition to any gender, vaccination coverage has to be very extensive and should cover very subgroup of the population. As there is no clear-cut idea on the proportion of population to be covered for attaining herd immunity and also the duration of antibody stay in the human body or effectiveness of cell-mediated immunity post-vaccination, it is advised to continue following preventive measures until a clearer picture of the current pandemic is established. Hence, the golden rule of Social distancing, hand sanitization, usage of personal protective equipment such as masks and implementation of Public health measures have to be continued.

\section{Recommendation}

Non-Pharmaceutical interventions with focus on High Risk Groups (HRG) need to be continued to further prevent further spread of infection.

Repeat survey may be planned to assess the effectiveness of vaccination program at different stages.

\section{Conflict of Interest: None}

\section{References}

1. Zhu N, Zhang D, Wang $W$ et al. A novel coronavirus from patients with pneumonia in China, 2019. N Engl J Med 2020; 382(8): 727-733.

2. World Health Organisation. WHO announces COVID-19 outbreak a pandemic. World Health Organisation. World Health Organization. 2020. p. 1. Available from: https://www.euro.who.int/en/health-topics/ health-emergencies/coronavirus-covid-19/news/ news/2020/3/who-announces-covid-19-outbreak-apandemic.

3. Worldometer. Coronavirus Cases. Worldometer. 2020. pp. 1-22. Available from: https://www.worldometers. info/coronavirus/.

4. Ministry of Health and Family Welfare. 2020. Available from: https://www.mohfw.gov.in/.

5. The Lancet. India under COVID-19 Lockdown. Lancet 2021; 395(10233): 1315.

6. Murhekar M, Bhatnagar T, Selvaraju S et al. SARS-CoV-2 Antibody prevalence in India: findings from the second Nationwide Household Serosurvey. 2020.

7. BBC News. India. Feb 5, 2021. ICMR sero survey: One in five Indians exposed to Covid-19. 2021. Available from: https://www.bbc.com/news/world-asiaindia-55945382.

8. Padhi A, Kumar S, Gupta E et al. Laboratory Diagnosis of Novel Coronavirus Disease 2019 (COVID-19) Infection. In: Coronavirus Disease 2019 (COVID-19). Nature Publishing Group 2020. 95-107.

9. To KKW, Tsang OTY, Leung WS et al. Temporal profiles of viral load in posterior oropharyngeal saliva samples and serum antibody responses during infection by SARS-CoV-2: an observational cohort study. Lancet Infect Dis 2020; 20(5): 565-574.

10. Guidance on developing a national deployment and vaccination plan for COVID-19 vaccines. 2020. Available from: https://www.who.int/publicationsdetail-redirect/WHO-2019-nCoV-Vaccine_ deployment-2020.1.

11. Jacofsky D, Jacofsky EM, Jacofsky M. Understanding antibody testing for Covid-19. The Journal of Arthroplasty 2020.

12. Wikramaratna $P$, Paton RS, Ghafari M et al. Estimating false-negative detection rate of SARS-CoV-2 by RT-PCR. medRxiv, 2020.

13. Li Z, Yi Y, Luo X et al. Development and clinical application of a rapid IgM-IgG combined antibody test for SARSCoV-2 infection diagnosis. J Med Virol 2020.

14. Kumar MS, Bhatnagar T, Manickam P et al. National sero-surveillance to monitor the trend of SARS-CoV-2 infection transmission in India: Protocol for communitybased surveillance. Indian Journal of Medical Research 2020; 151(5): 419.

15. Jabalpur District: Census 2011-2020 data. 2021. Available from: https://www.census2011.co.in/census/ district/318-jabalpur.html.

16. Zimmermann $P$, Curtis N. Coronavirus infections in children including COVID-19: an overview of the epidemiology, clinical features, diagnosis, treatment and prevention options in children. The Pediatric Infectious Disease Journal 2020; 39(5): 355.

17. Kelvin AA, Halperin S. COVID-19 in children: the link in the transmission chain. The Lancet Infectious Diseases, 2020.

18. Ministry of Rural Development. Poverty measurement in India. Tendulker Committee for BPL. 2021. Available from: https://rural.nic.in/sites/default/files/ WorkingPaper_Poverty_DoRD_Sept_2020.pdf.

19. Shakiba M, Nazari SS, Mehrabian F et al. Seroprevalence of COVID-19 virus infection in Guilan province, Iran. medRxiv, 2020.

20. Rosenberg ES, Tesoriero JM, Rosenthal EM et al. Cumulative incidence and diagnosis of SARS-CoV-2 infection in New York. medRxiv, 2020.

21. Streeck H, Hartmann G, Exner M et al. Vorläufiges Ergebnis und Schlussfolgerungen der COVID-19 CaseClusterStudy (Gemeinde Gangelt)[Preliminary results and conclusions from the COVID-19 case-clsuter study (community Gangelt).

22. Media Bulletin. Hindustan Times. 2021. Available from: https://www.hindustantimes.com/india-news/ high-exposure-to-covid-19-in-urban-areas-find-serosurveys/story-sRzruWmP3qUZNz1BXnOC3I.html.

23. Media Bulletin. The Hindu. 2020. Available from: 
https://www.thehindu.com/news/cities/Delhi/delhissero-prevalence-study-finds-2348-per-cent-peopleaffected-by-covid-19/article32147726.ece.

24. Sakalle S, Saroshe S, Shukla H et al. Seroprevalence of antiSARSCoV2 antibodies in Indore, Madhya Pradesh: A community-based crosssectional study. J Family Med Prim Care 2020.

25. Stringhini S, Wisniak A, Piumatti G et al. Seroprevalence of anti-SARS-CoV-2 IgG antibodies in Geneva, Switzerland (SEROCoV-POP): a population-based study. The Lancet 2020. 